\title{
Novel pulsed electron spin resonance system and studies of phosphorus in natural silicon
}

\author{
N. Suwuntanasarn • W. D. Hutchison • \\ G. N. Milford · R. Bramley
}

Published online: 8 August 2008

(C) Springer Science + Business Media B.V. 2008

\begin{abstract}
An X-band pulsed electron spin resonance system has been designed and constructed specifically for studies of decoherence times of phosphorus donor electrons in silicon. The microwave electromagnetic field aspects of the structure have been analysed, and the probe head geometry optimised, through 3D electromagnetic simulations. Results for natural silicon samples at temperatures down to $6 \mathrm{~K}$, are analysed and we obtain an estimate of the isolated spin decoherence time in ${ }^{\text {nat }} \mathrm{Si}$ approximately an order of magnitude longer than previously measured.
\end{abstract}

Keywords Electron spin echo $\cdot$ Decoherence time $\cdot$ Phosphorus in silicon

\section{Introduction}

Phosphorus donor electrons in silicon (Si:P) are reported to have long (both nuclear and electron) spin relaxation times [1], and in addition, its compatibility with existing device fabrication technology, makes it as a potential candidate for a quantum computing (QC) device [2]. In Si:P the dephasing of the donor electron spin represents the decoherence time of the device (single qubit decoherence). Pulsed electron spin resonance (ESR) offers a convenient and most effective way to study this dephasing

N. Suwuntanasarn · W. D. Hutchison $(\bowtie)$

Centre for Quantum Computer Technology, School of Physical,

Environmental and Mathematical Sciences, UNSW@ADFA,

Canberra, ACT 2600, Australia

e-mail:w.hutchison@adfa.edu.au

G. N. Milford

Centre for Quantum Computer Technology, School of Information Technology and Electrical

Engineering, UNSW@ADFA, Canberra ACT 2600, Australia

R. Bramley

Research School of Chemistry, The Australian National University,

Canberra, ACT 0200, Australia 
mechanism. General pulsed ESR studies of Si:P were conducted a long time ago $[3,4]$, and recently [5-8] after the Si:P proposal for the use as a QC device [2]. Therefore, an X-band pulsed ESR system has been set up to provide the most optimal conditions for the Si:P decoherence time measurement. The system includes a resonator with high conversion efficiency factor, $\Lambda\left(\Lambda=B_{1} /(P Q)^{0.5}\right)$. Details of the design were presented in [9]. This paper details a low temperature, $T$, experimental verification of the system through the electron spin echo (ESE) measurement of ${ }^{n a t} \mathrm{Si}: \mathrm{P}$. We report a decoherence time in ${ }^{\text {nat }} \mathrm{Si}$ that is approximately an order of magnitude longer than previously seen [8].

\section{Pulsed ESR system}

The probe head for the system, which can be easily fitted within a CW-ESR cryostat, consists of a specially designed resonator, plus microwave ( $\mathrm{mw}$ ) and cryostat shields with an adjustable mechanism for tuning and matching purposes, and a Gordon coupler to provide coupling via a rectangular iris. The whole probe head structure was optimised with the aid of the Computer Simulation Technology (CST) package [10] and maximises the conversion factor. The resonator is a two-gap rectangular loop gap resonator (R-LGR), which is particularly suitable for a flat sample type. The probe head allows both mw electric $(\mathrm{E})$ field and magnetic $(\mathrm{H})$ field couplings to the resonator, hence providing the advantage for sample orientation dependent decoherence time measurement. The $\mathrm{mw} \mathrm{E}$ and $\mathrm{H}$ field distributions of the resonators simulated (Fig. 1) show that a silicon wafer located inside the resonator experiences the maximum mw $\mathrm{H}$ field, and the minimum mw E field. The probe head functionality was verified experimentally via a network analyser measurement and ESE experiments. An example of the $\mathrm{S}_{11}$ captured from the network analyser agrees well with simulations (Fig. 1). The simulated $\Lambda$ of the probe head is $\sim 0.012 \mathrm{mT} / \sqrt{ } \mathrm{W}$.

\section{Experimental results and discussion}

${ }^{n a t}$ Si:P samples with low $\mathrm{P}$ concentrations of $7.5 \times 10^{14} \mathrm{P} / \mathrm{cm}^{3}$ were studied since the decoherence time measurement has the least effect from donor-donor interactions. Indeed, the dominant electron spin dephasing mechanism is the spectral diffusion (SD) from its interaction with the non-zero silicon nuclear spin (Si-29, 4.7\% in natural silicon). Here the ESE amplitude of nat $\mathrm{Si}: \mathrm{P}$ were recorded as a function of inter-pulse separation, $\tau$, with the $\pi / 2-\tau-\pi$-echo sequence, where $\pi / 2$ is $16 \mathrm{~ns}$ and $\pi$ is 32 ns respectively. Temperature, $T$, is carefully controlled as the decoherence time is strongly temperature dependent. Two-pulse ESE measurements (Fig. 2) were conducted at two different $B_{0}$-field-to-sample crystal orientations, $\Theta\left(\Theta=0^{\circ}\right.$ when $B_{0} \|[100]$ and $\Theta=90^{\circ}$ when $B_{0} \|[110]$ at $8 \mathrm{~K}, 7 \mathrm{~K}$ and $6.3 \mathrm{~K}$.

The echo decay results are fitted with $V(\tau)=V_{0} \exp \left[-\left(2 \tau / T_{\mathrm{M}}\right)-\left(2 \tau / T_{\mathrm{SD}}\right)^{3}\right]$ where $T_{\mathrm{M}}$ is the exponential decay time constant, which incorporates a number of processes mentioned later, $T_{\mathrm{SD}}$ is the SD decay time, $n$ is an exponential stretching factor, varied between 2 and 3 for different SD regimes [6]. $T_{M}$ results fitted by the relationship above are given in Table 1. Other ESE experiments were performed by varying the second pulse turning angle, $\theta$. It was found that $T_{\mathrm{M}}$ increased as $\theta$ 

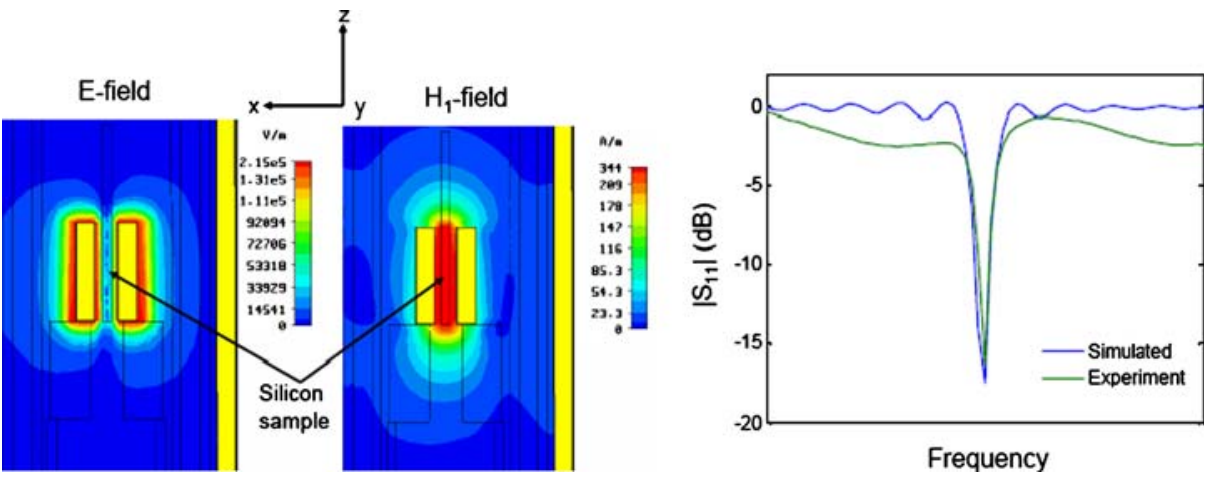

Fig. 1 The simulated and experimental results on the probe head structure. The simulated results on the microwave $\mathrm{E}$ and $\mathrm{H}$ field distribution shown a $\mathrm{Z}-\mathrm{X}$ cross sectional plane (left). A silicon sample is located in the centre of the resonator (right). A comparison of the $\mathrm{S}_{11}$ results for the E-field coupled resonator at the coupling distance $=0 \mathrm{~mm}$ from the network analyser and the simulations

Fig. 2 The two-pulse echo decay in ${ }^{\text {nat }} \mathrm{Si}: \mathrm{P}\left(7.5 \times 10^{14}\right.$ $\mathrm{P} / \mathrm{cm}^{3}$ ) at different temperatures, $T$, and sampleto-static magnetic field $\left(B_{0}\right)$ orientations, $\Theta$

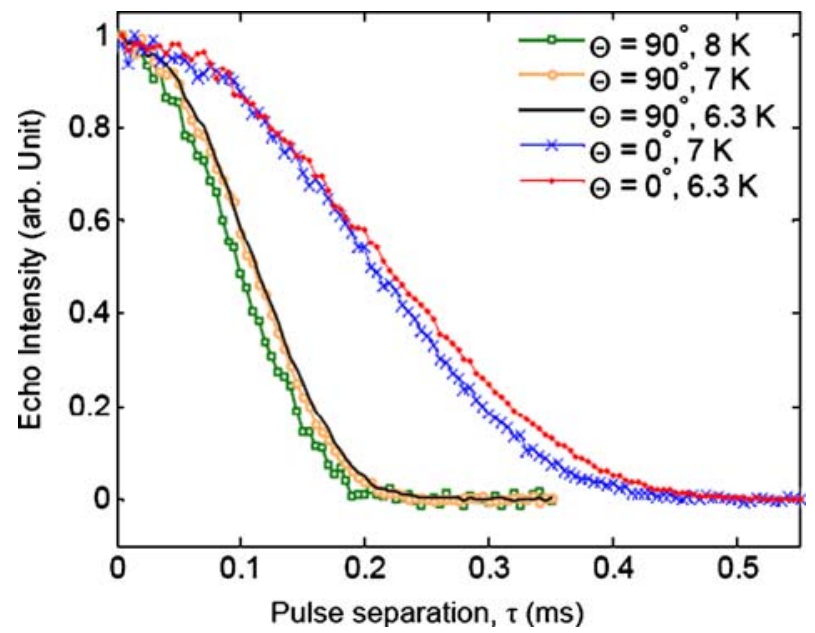

Table 1 Results of the fitting of the echo decay of ${ }^{\text {nat }} \mathrm{Si}: \mathrm{P}$ at $7.5 \times 10^{14} \mathrm{P} / \mathrm{cm}^{3}$ at 8,7 and $6.3 \mathrm{~K}$

\begin{tabular}{lllllll}
\hline Setup & $T(\mathrm{~K})$ & $T_{\mathrm{M}}(\mathrm{ms})$ & $T_{\mathrm{m} 0}(\mathrm{~ms})$ & {$[\mathrm{P}]=2 C$} & $T_{\mathrm{SD}}(\mathrm{ms})$ & $n$ \\
\hline$\Theta=0^{\circ}$ to $[100]$ & 6.37 & $1.6 \pm 0.2$ & $3.1 \pm 0.4$ & $7.0 \pm 0.5$ & $0.58 \pm 0.05$ & $2.8 \pm 0.2$ \\
& & $1.2 \pm 0.2$ & $2.2 \pm 0.4$ & $8.5 \pm 0.5$ & $0.56 \pm 0.05$ & $3.1 \pm 0.2$ \\
$\Theta=90^{\circ}$ to $[100]$ & 6.3 & $1.8 \pm 0.2$ & $3.4 \pm 0.4$ & $6.0 \pm 0.5$ & $0.28 \pm 0.04$ & $2.7 \pm 0.2$ \\
& 7 & $1.3 \pm 0.2$ & $2.3 \pm 0.4$ & $8.4 \pm 0.5$ & $0.28 \pm 0.04$ & $2.9 \pm 0.2$ \\
& 8 & $0.8 \pm 0.2$ & $1.1 \pm 0.4$ & $7.6 \pm 0.5$ & $0.26 \pm 0.05$ & $2.9 \pm 0.2$ \\
\hline
\end{tabular}

decreased, and as such indicated that the instantaneous diffusion (ID) contributes significantly to the observed $T_{\mathrm{M}}$. The usual method to find the intrinsic phase memory time, $T_{\mathrm{m} 0}$, also defined as a decoherence time of an isolated electron spin free from the effect of ID is by extrapolating the second pulse turning angle, $\theta$ to zero. However instead, we follow a procedure introduced by Ferretti et al. [8] where 
$T_{\mathrm{m} 0}$ is derived from $T_{\mathrm{M}}$ and the ID time, $T_{\mathrm{ID}}$ via the relationship $1 / T_{\mathrm{M}}=1 / T_{\mathrm{m} 0}+$ $1 / T_{\mathrm{ID}}$. Where $T_{\mathrm{ID}}$ is approximated from $1 / T_{\mathrm{ID}}=C \pi \mu g^{2} \mu_{\mathrm{B}}^{2} \sin ^{2}(\theta / 2) /(9 \sqrt{ } 3 \hbar)$ where $C$ is the concentration of the excited electron spins (while $\mathrm{P}$ concentration in a sample, $[\mathrm{P}]=2 C), \mu$ is the permeability of crystalline silicon, $g$ is the $g$-factor of the donor electron, $\mu_{\mathrm{B}}$ is the Bohr magneton. The echo decay is also set as a function of $\theta$ and the $T$, so the relationship can be re-written as:

$$
V(\tau, \theta, T)=V(0, \theta, T) \exp \left[-2 \tau\left(\frac{1}{T_{\mathrm{m} 0}(T)}+\frac{1}{T_{\mathrm{ID}}(\theta)}\right)-\left(\frac{2 \tau}{T_{\mathrm{SD}}(T)}\right)^{n}\right]
$$

The results of the echo decay fitted by Eq. 1 are also reported in Table 1.

The results of Fig. 2 (and Table 1) have shown a number of interesting points. Firstly, the echo decay is strongly $\Theta$ dependent and non-single exponential, as a result of variations in the SD term. $T_{\mathrm{SD}}$ values fitted from both equations were found to be independent of $T$, but strongly dependent on $\Theta$. Secondly, the results show the trend that $T_{\mathrm{M}}$ and $T_{\mathrm{m} 0}$ increase as $T$ decreases, regardless of $\Theta$ or fitting method. The value of $[\mathrm{P}]$ determined from the fitting is also reasonably close to the actual value. It was found that no matter which fitting method is used, the trend always shows that longer decoherence time was obtained at lower $T$ (also noticeable from the echo decay in Fig. 2). Moreover, our $T_{\mathrm{M}}$ and $T_{\mathrm{m} 0}$ obtained at $7 \mathrm{~K}$ are an order of magnitude longer than results from a sample with approximately an order of magnitude higher $\mathrm{P}$ concentration $T_{\mathrm{M}} / T_{\mathrm{m} 0}=0.092 / 0.22 \mathrm{~ms}$ respectively [8], indicating that ID is still a major contribution to the observed echo decay. This behaviour was also observed in isotropically pure silicon $[5,6]$ and since the expected ID rate $\left(1 / T_{\mathrm{ID}}\right)$ is linearly proportional to the doping density, even longer decoherence times should be achievable at lower concentrations. Therefore, longer decoherence times could be achieved by lowering the temperature (as seen in this report), lowering concentration (derived from the relationship) or lowering the non-zero nuclear spin of silicon as reported by $[5,6]$.

\section{References}

1. Feher, G., Gere, E.A.: Electron spin resonance experiments on donors in silicon II: electron spin relaxation effects. Phys. Rev. 114, 1245-1256 (1959)

2. Kane, B.E.: A silicon-based nuclear spin quantum computer. Nature 393, 133-137 (1998)

3. Gordon, J.P., Bowers, K.D.: Microwave spin echoes from donor electrons in silicon. Phys. Rev. Lett. 1, 368-370 (1958)

4. Chiba, M., Hirai, A.: Electron spin echo decay behaviours of phosphorus doped silicon. J. Phy. Soc. Jpn. 33, 730-738 (1972)

5. Tyryshkin, A.M., Lyon, S.A., Astashkin, A.V., Raitsimring, A.M.: Electron spin relaxation of phosphorus donors in silicon. Phys. Rev. B 68, 193207 (2003)

6. Tyryshkin, A.M., Morton, J.J.L., Benjamin, S.C., Ardavan, A., Briggs, G.A.D., Ager, J.W., Lyon, S.A.: Coherence of spin qubits in silicon. J. Phys.: Condens. Matter 18, S783-S794 (2005)

7. Abe, E., Itoh, K.M., Isoya, J., Yamasaki, S.: Electron-spin phase relaxation of phosphorus donors in nuclear-spin-enriched silicon. Phys. Rev. B 70, 33204 (2004)

8. Ferretti, A., Fanciulli, M., Ponti, A., Schweiger, A.: Electron spin-echo relaxation and envelope modulation of shallow phosphorus donors in silicon. Phys. Rev. B 72, 235201 (2005)

9. Suwuntanasarn, N., Hutchison, W.D., Milford, G.N., Bramley, R.: A novel probe head for an electron spin echo of an Australian coal. In: Proceedings of the 31st Annual ANZIP Condensed Matter and Materials Meeting, 93-95 (2007)

10. CST.: Http://www.cst.com. cited 20 Jul 2007 (2007) 